\title{
Cuba's National Regulatory Authority \& COVID-19: Olga Lidia Jacobo-Casanueva MS Director, Center for State Control of Medicines and Medical Devices (CECMED)
}

Tania L. Aguilar-Guerra MD MS, Esther M. Fajardo-Díaz MS, Conner Gorry MA

As of this writing, more than 4.6 million Cubans (over $40 \%$ of the population on the island), had received at least their first dose of Soberana 02 or Abdala, two of five vaccine candidates for SARS-CoV-2 developed and produced on the island. Late-phase clinical trial data revealed that Abdala is $92.28 \%$ effective after the full, three-dose cycle and Soberana 02 is $91.2 \%$ effective after two doses, when followed by a booster of Soberana Plus.[1] Cuban health authorities have committed to vaccinating the entire population, including children aged 3-18 years old, using these vaccines by the end of 2021. The first pre-clinical, peer-reviewed data are available,[2] with clinical trial results already submitted to various international journals.

Building on decades of biotechnology know-how developing, producing and administering 11 preventive vaccines for childhood diseases-used in the nation's universal health system and also marketed elsewhere-Cuba is the first, and to date only, country in Latin America and the Caribbean to develop its own vaccine candidates for COVID-19 (Soberana 01; Soberana 02; Soberana Plus; Abdala and Mambisa; see Box on following page). In a strategy designed to ensure comprehensive and importantly, independent solutions to the global health crisis, research institutes and manufacturing facilities coordinated by BioCubaFarma-the country's biopharmaceutical conglomerate-have also developed COVID-19 treatments and essential medical equipment.

To gain a better understanding of the regulatory process involved, MEDICC Review turned to Olga Lidia JacoboCasanueva, Director of the Center for State Control of Medicines and Medical Devices (CECMED), Cuba's national regulatory authority (NRA). A clinical microbiologist, JacoboCasanueva served as interim director throughout 2020 before becoming director in January 2021. She has spent nearly her entire career at CECMED, working her way up the ranks in a unique trajectory: from her first position in 1992 in the Center's microbiology laboratories, she has since worked in all but one of the six areas required by WHO to qualify as a National Regulatory Authority of Reference (NRAr; CECMED was certified as a Level 4 NRAr in 2011, a qualification it maintains). In short, Jacobo-Casanueva is a regulatory polymath, with

MEDICC Review: Can you synthesize CECMED's role as a national regulatory authority?

Olga Lidia Jacobo: Any medicine, device or equipment-Cuban or imported-used in our health system must be approved by CECMED, the country's single regulatory authority. To ensure new products meet global standards of quality, safety and efficacy, WHO defines the six basic functions for NRAs as: medicine regis-

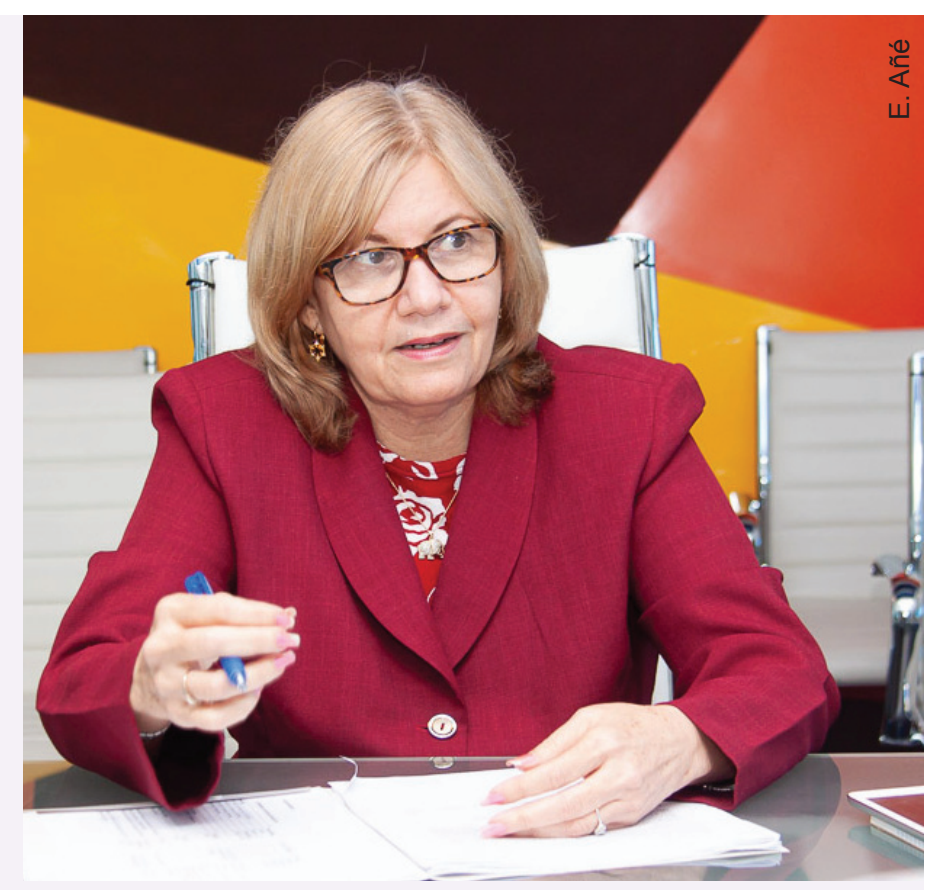

hands-on experience in nearly every facet of regulation. She is also an adjunct researcher in the Faculty of Biology at the University of Havana.

Cuba's decision to confront the pandemic autonomously by developing preventive vaccines to control COVID-19 is deliberate and fraught with challenges. With dozens of ongoing clinical trials, coupled with the declining epidemiological and economic situation in Cuba-exacerbated by tightened US sanctions affecting all facets of COVID-19 prevention and response-we appreciate the time Jacobo-Casanueva took from her schedule to parse the complex regulatory mechanisms required to introduce Cuban and imported products into the national health system.

Editor's note: Just days after this interview was conducted in Havana, CECMED granted Emergency Use Authorization for Abdala, one of five Cuban COVID-19 vaccine candidates undergoing clinical trials since 2020. 
under the leadership of Dr Rafael Pérez-Cristiá, was restructured in $\mathbf{2 0 0 0}$ to conform to international standards. My first job at CECMED was to organize the lot-release system for vaccines in the newly-established biologicals division. Once we designed the system, we began implementing it with Cuba's meningococcal $B C$ vaccine (VAMENGOC-BC) and the recombinant hepatitis $B$ vaccine (Heberbiovac $\mathrm{HB}$ ). We then set to work on re-organizing and improving other functions falling within CECMED's purview including inspections and medicine registration specific to vaccines. With the restructuring in place, we turned our sights to systematizing the remaining NRA functions.

Our researchers continued to innovate and develop novel vaccines and therapies-making CECMED's efforts to comply with international guidelines paramount. This was a priority leading up to $\mathrm{WHO}$ inspections and pre-qualification for the Genetic Engineering and Biotechnology Center's (CIGB) recombinant hepatitis $B$ vaccine. Obtaining $\mathrm{WHO}$ pre-qualification for vaccines is an arduous, biennial process, designed to ensure the regulatory authority, production facilities and research laboratories are qualified to evaluate, register and control the entire manufacturing process.

\section{MEDICC Review: WHO pre-qualification for that Cuban vaccine ushered in an era of closer collabo- ration among Cuba, PAHO/WHO and countries in the region, correct?}

Olga Lidia Jacobo: That's right. CECMED and CIGB passed the inspections, the recombinant hepatitis $B$ vaccine received pre-qualification and our work in the region around regulation intensified. Since then, our specialists have worked very closely with $\mathrm{PAHO} /$ WHO and other countries in the region to develop biological standardization guidelines, advise on technical documents and serve as experts within thematic work groups. The idea is that specialists from the eight countries with Level 4 NRArs in the Americas (Argentina, Brazil, Canada, Chile, Colombia, Cuba, Mexico and the United States) help develop guidelines and provide expertise for countries with less-developed regulatory authorities.

For example, CECMED has been part of the Pan American Network for Drug Regulatory Harmonization (PANDRH), since its formation in 1999. Within this network are groups of specialists organized by theme; I coordinated the vaccines working group for the Americas and we drafted the common technical document (CTD) establishing requirements for vaccine registration. This document, Harmonized requirements for the licensing of vaccines in the Americas and Guidelines for preparation of application (https://iris. paho.org/handle/10665.2/31215), was approved and now is a tool any regulatory authority in the region can use. Coordinating this was a wonderful experience allowing me to work with specialists from throughout the hemisphere.
Clinical Trials Timeline for Cuban COVID-19 Vaccine Candidates

\section{Event} Date

\section{0}

CECMED authorizes parallel phase $1 / 2$ clinical trials for

Soberana 01

Soberana 01 phase $1 / 2$ clinical trials begin in Havana

CECMED authorizes phase 1 clinical trials for Soberana 01A

CECMED authorizes phase 1 clinical trials for Soberana 02

Phase 1 Soberana 02 clinical trials begin (Havana; 40 volunteers)

CECMED authorizes parallel phase $1 / 2$ clinical trials for Abdala

CECMED authorizes parallel phase $1 / 2$ clinical trials for Mambisa

Phase 1 Mambisa clinical trials begin (Havana; 88 volunteers)

Phase 1 Abdala clinical trials begin (Santiago de Cuba; 132

volunteers)

CECMED authorizes phase 2 clinical trials for Soberana 02A

\section{1}

CECMED authorizes phase 1 clinical trials for Soberana 01B

Phase 2 Soberana 02 clinical trials begin (Havana; 810

volunteers)

Phase 2 Abdala clinical trials begin (Santiago de Cuba; 660

volunteers)

CECMED authorizes phase 3 clinical trials for Soberana 02

Phase 3 clinical trials begin (Havana; 44,010 volunteers)

CECMED authorizes phase 3 clinical trials for Abdala

CECMED authorizes health intervention study for Soberana 02

Soberana 02 health intervention study begins (Havana; 150,000 volunteers)

Phase 3 Abdala clinical trials begin (Santiago de Cuba, Granma, Guantánamo Provinces; 48,000 volunteers)

CECMED authorizes health intervention study for Abdala

March 22

Abdala health intervention study begins (Santiago de Cuba; Granma; Guantánamo Provinces; 120,000 volunteers)

CECMED authorizes phase 2 clinical trial for Soberana Plus in COVID-19 convalescent patients

Soberana public health intervention begins; Abdala public health intervention begins

CECMED authorizes Soberana Pediatría, parallel phase 1/2 trials for Soberana 02 in children

Soberana Pediatría phase 1/2 trials begin (Havana; 50

volunteers ages 3-18)

CECMED authorizes Ismaelillo, parallel phase 1/2 trials

for Abdala in children

August 13

August 24

October 17

October 27

November 2

November 26

November 27

December 7

December 7

December 17

January 5

January 18

February 1

March 3

March 4

March 18

March 19

March 22

March 27

March 29

April 9

May 12

June 10

June 14

July 1

CECMED issues Emergency Use Authorization (EUA) for Abdala July 9

CECMED authorizes parallel phase $1 / 2$ clinical trials for Abdala and Mambisa combination vaccine in COVID-19 convalescent July 9 patients

Soberana Pediatría phase 2 trial begins (Havana; 300 volunteers ages 3-18)

July 14

Ismaelillo phase 1/2 trials begin (Camagüey; 592 volunteers ages 3-18)

July 15

CECMED authorizes Soberana Centro, parallel phase 2

non-inferiority clinical trials

July 23

Sources: http://www.cubadebate.cu/especiales/2021/03/30/cuba-en-datos-el-camino-hacia -la-inmunizacion/

https://www.cubahora.cu/blogs/datos-con-sentido/candidatos-vacunales-cubanos-lo-que -debes-saber

https://salud.msp.gob.cu/actualizacion-de-la-vacunacion-en-el-marco-de-los-estudios-de-los -candidatos-vacunales-cubanos-y-la-intervencion-sanitaria/

https://rpcec.s/d.cu/ 
More recently, we consulted on the forthcoming document establishing a framework for evaluating national regulatory authorities globally. To be included in this framework requires an extraordinarily rigorous evaluation and inspection process, which CECMED is scheduled to undergo at the end of 2021. This is a complex endeavor, with module-by-module evaluations, but we have to buckle down and prepare so we can demonstrate the rigor of our regulatory authority, on par with others around the world.

MEDICC Review: With the COVID-19 pandemic, vaccine regulatory mechanisms are being closely scrutinized-a topic covered extensively in previous issues of our journal. [3] Can you provide examples of how CECMED adapted and streamlined its process?

Olga Lidia Jacobo: Normally a vaccine takes between five and ten years from development to licensing, but the urgency of the pandemic required regulatory authorities to adjust processes to make them more agile, without compromising science or regulatory best practices. In Cuba, we implemented a streamlined, collaborative process that has proven fundamental in shortening the clinical trial timeline while maintaining international standards. Under normal circumstances, a vaccine producer submitted to CECMED all necessary documentation, protocols, etc., to apply for clinical trial authorization. This was a very slow process because we would usually make recommendations to bring the design into line with international best practices or to otherwise improve the rigor of the trial. For this reason, we were often criticized for being too slow, but CECMED is the ultimate authority, with the highest level of responsibility — we are very meticulous and demanding as a result.

When COVID-19 was declared a global health crisis in early 2020, the Cuban Ministry of Public Health (MINSAP) convened the Innovation Committee, a multi-disciplinary work group including scientists, specialists, biopharmaceutical and technological experts and others. CECMED, in our capacity as independent regulatory agency, is also part of the Committee, though our role has not changed: we remain a non-partisan judge of clinical trial designs, ensuring their compliance with global regulatory norms.

The Innovation Committee strategy pivots on debating which products and devices should be prioritized and rather than wait for the manufacturer to submit their final trial design for authorization-which often would require changes and slow down the process-CECMED now makes recommendations earlier, limiting the back and forth and avoiding re-design of trials. We made other adjustments as well, to timeframes around pre-clinical trials and stability studies for instance, but also strengthening our surveillance system, especially around adverse events following immunization (AEFI). As an NRAr, these adaptations had to be made within a legal framework. As always, scientific rigor, proper documentation and a legal foundation drive our strategy. Since the beginning of the pandemic, CECMED has issued 13 regulatory provisions providing a legal basis to make these adjustments.

MEDICC Review: Cuba's COVID-19 vaccine candidates were developed rapidly and completed several clinical trial phases in 2020 and 2021. How close is CECMED to issuing Emergency Use Authorization (EUA) for these vaccines?

Olga Lidia Jacobo: We are right now evaluating the CTD and conducting inspections for the Abdala vaccine submitted for this purpose. This isn't just a question of looking over some paperwork. We have to evaluate all documentation related to the vaccine, including pre-clinical data, toxicology and animal studies, plus clinical trial results including safety and immunogenicity data, statistical analysis and more. Analyzing vaccine quality is also part of CECMED's work in terms of evaluating the entire production process for consistency, lot production, best practices compliance within the manufacturing facility and validity of the technical analyses.

Our role also includes conducting inspections throughout every phase of trials to ensure compliance with protocols and best practices. This means we visit every vaccination site including hospitals, community polyclinics and family doctor-and-nurse offices where vaccinations are administered. We also verify that the cold chain is properly maintained-if vaccines are distributed at 5:00 AM, CECMED specialists are there before then, and also at the vaccination sites to measure the temperature upon arrival to ensure there has been no break in the cold chain. We also inspect the transportation mechanisms and protocols of the Medicines Marketing and Distribution Company (EMCOMED) responsible for delivering the vaccines.

\section{MEDICC Review: How is it possible that Cuba vaccinated over 2 million people without having first obtained EUA for either of its two vaccines in phase 3 clinical trials?}

Olga Lidia Jacobo: The fact that Cuba launched intervention studies in high-risk groups (health and BioCubaFarma workers, followed by a public health intervention in the hardest-hit Havana municipalities that were the epicenter of the pandemic at that time) before receiving EUA has been a topic of huge debate. It's important to note that CECMED, along with BioCubaFarma, which represents the pharmaceutical industry, are independent entities, but also sit on the Innovation Committee that made that decision. It wasn't made in a vacuum, but rather using the data we had at hand. And we had a lot of data at that pointfrom completed phase 1 and 2 trials and ongoing phase 3 trials, after many doses had already been administered with only mild adverse events. So we had data demonstrating the safety and immunogenicity of Soberana 02 and Abdala at this point. [The public health intervention was initiated after over 145,000 Cubans had received their full vaccinations using one of these two candidates in clinical trials and the intervention study, Eds].

At that moment, Cuba was facing a very complex epidemiologic scenario, with variants of concern circulating in the country and high rates of autochthonous transmission. So the Innovation Committee convened, with CECMED presenting the vaccine data to date, epidemiologists presenting transmission data and virologists from the Pedro Kourí Tropical Medicine Institute (IPK) providing the latest information on variants of concern. Although these vaccines still had not received EUA, a costbenefit analysis concluded that the benefits outweighed the risks of a public health intervention, plus we had safety, immunogenicity and partial efficacy results. 
Let me also point out that while phase 3 clinical trials were not yet complete, trial protocols for these vaccines allowed for precisely this scenario. Used by vaccine producers around the world and known as a data cutoff date, it permits specialists to analyze vaccine efficacy using data at a defined point in the trial. The data cutoff date for both Soberana 02 and Abdala was 15 days after receiving two doses of the former and three doses of the latter (Abdala has a shorter vaccination schedule than Soberana 02-every 14 days versus every 28).

MEDICC Review: What role does WHO play in vaccine approval? And other regulatory authorities in countries seeking access to Cuban vaccines?

Olga Lidia Jacobo: WHO is an international organization that makes recommendations and supports national regulatory authorities but it does not approve vaccines; it isn't a regulatory authority itself. Once COVID-19 was declared a Public Health Emergency of International Concern (PHEIC), WHO began implementing streamlined mechanisms for faster delivery of vaccines to those countries needing them. One piece of this is a more agile authorization tool for vaccines known as Emergency Use Listing (EUL). WHO-recommended vaccines including Pfizer, Moderna and others, were authorized under this EUL category, following approval by the Federal Drug Administration (FDA) or the national regulatory agency of the country producing the vaccine. The process for Cuban vaccines is the same.

WHO Emergency Use Listing (EUL) is a procedure for assessing unlicensed vaccines, therapeutics and in vitro diagnostics during public health emergencies with the ultimate goal of expediting the availability of these products to people who need them. When products are not licensed yet (still in development), WHO will assess the quality, safety and efficacy (or performance) data generated during development and conduct a risk-benefit assessment to decide if they can be used outside clinical trials.

Source: WHO. Coronavirus disease (COVID-19): Use of Emergency Use Listing procedure for vaccines against COVID-19, 30 Sep 2020. https://www.who.int/news-room/q-a-detail/coronavirus-disease-use-of -emergency-use-listing-procedure-forvaccines-against-covid-19

Soberana 02 and Abdala have not yet received our own EUA, required for EUL. Our regulatory process is based on reviewing all the technical documentation for these vaccines, plus inspections of production facilities, fundamental for certifying best practices throughout the process. National regulatory authorities interested in introducing Cuban vaccines into their health systems-Mexico, Argentina and other countries beyond Latin America have signed collaboration agreements with Cuba-will apply their regulatory process to our vaccines before they are administered. CECMED has been working closely with $\mathrm{PAHO}$ to foster cooperation and transparency among national regulatory authorities and promote Good Regulatory Practices (GRP) and Good Reliance Practices (GReP) in the region so that when a vaccine receives EUA, the approval process can proceed quickly and confidently by individual NRAs in countries wishing to administer it.

\section{MEDICC Review: Will CECMED have a lighter work load once the EUA is issued?}

Olga Lidia Jacobo: Not at all. There's the entire vaccine licensure process that is usually quite long but will probably happen fairly quickly since these vaccines have amassed so much data already. And two areas of regulation are ongoing: vaccine lot release (every lot of vaccine produced must be analyzed and authorized by CECMED specialists) and postmarketing surveillance, which is critically important.

CECMED is also responsible for evaluating and authorizing the use of any medical device or equipment used in the national health system-like ventilators and SARS-CoV-2 test kits, for example. We work closely with various BioCubaFarma companies that manufacture medical equipment and once they started developing ventilators, we assumed our role as regulatory authority; after the corresponding pre-clinical and clinical trials, one of these ventilators was approved for emergency use, according to clinical protocols, in patients meeting specific criteria. All imported medicines, meanwhile, plus medical devices and equipment, are subject to CECMED oversight and authorization, as well. In short, anything entering the country for use in the health system-whether purchased or donated-has to be licensed and authorized by CECMED to ensure proper control and surveillance.

And then there are all the other ongoing clinical trialseverything from drug repositioning and treatments to COVID-19 pediatric vaccines.

MEDICC Review: You mention drug repurposing or repositioning, which has been a strategy employed widely in the search for COVID-19 treatments. Is Cuba pursuing this? What is CECMED's role in this scenario?

The severity of the
pandemic-with so many
people falling gravely ill
and dying-prompted
us to think about drug
repositioning early on

Olga Lidia Jacobo: The severity of the pandemicwith so many people falling gravely ill and dyingprompted us to think about drug repositioning early on. Several likely candidates for repositioning were presented to the Innovation Committee, activating CECMED's role as regulatory authority. This is how Jusvinza and Itolizumab entered into Cuban treatment protocols.

Jusvinza (produced by CIGB), is a peptide that while still not registered, has undergone clinical trials for treating rheumatoid arthritis. Specialists hypothesized that the effect this peptide has on the cytokine storm and hyperinflammation might be useful in treating seriously ill COVID-19 patients. They presented data from the rheumatoid arthritis trials and argued for compassionate use authorization-also known as expanded access_-of Jusvinza for certain patients. This type of authorization falls within CECMED's legal framework and is used around the world for patients with life-threatening conditions when no other therapeutic options are available. Itolizumab (produced by the Molecular Immunology Center, CIM), a monoclonal antibody, also acts to mitigate hyperinflammation and was developed to treat severe psoriasis and cutaneous 
T-cell lymphoma. This is another Cuban product that received expanded-access authorization for use in our health system; it is in clinical trials overseas specifically for treating COVID-19, and more data will be available soon.

Another Cuban product, Biomodulina $\mathrm{T}$ (produced by the National Biopreparations Center, BioCen), is an immunomodulator that supports immune system response; it is registered and approved for use in our health system for patients with recurrent respiratory infections. Since it underwent clinical trials, is legally registered and boosts the immune system, some had hoped we could just start treating COVID-19 patients with Biomodulina T. But regulation doesn't work that way. To treat a different condition-in this case COVID-19-it must go through the entire authorization process as if it were a new drug, with separate clinical trials designed for treating this disease and official, unique registration in the Cuban Public Registry of Clinical Trials.

MEDICC Review: There has been a troubling surge in pediatric COVID-19 cases globally, but also in Cuba. You mentioned clinical trials specifically for children using Cuban vaccines...

Olga Lidia Jacobo: First let me say that we are even more demanding when it comes to pediatric vaccine trials: the immune system of a child is not the same as an adult, so we always perform clinical trials in the adult population first. COVID-19 vaccines are no different. The pediatric clinical trial administers Soberana 02 (2 doses, 0-28 days) followed by a Soberana Plus booster shot on day 56. A full description of this Soberana Pediatría clinical trial is available at: https://rpcec.sld .cu/en/trials/RPCEC00000374-En

\begin{tabular}{|l} 
Pediatric clinical trials \\
require even more rigor \\
due to the ethical \\
considerations involved
\end{tabular}

Pediatric clinical trials require even more rigor due to the ethical considerations involved. Understanding what the trial entails is part of it, but providing written, informed consent is indispensable as well. In the case of children under 12, parents or legal guardians sign the informed consent, but children and teens pre-selected for the trial have the right to say 'no, I don't want to participate.' Obviously, we all want our children to be vaccinated, so they can begin their school year and resume normal lives, but parents cannot obligate them to participate in the trial.

CECMED approved parallel phase 1/2 trials, now underway, at the Juan Manuel Márquez Pediatric Teaching Hospital in Havana. This hospital has extensive experience conducting clinical trials, including the requisite physical infrastructure, trained personnel and specialists, and adherence to clinical best practices. The first group of 25 youngsters, aged 12-18 years, received their first dose several weeks ago and were monitored for adverse effects for one week after vaccination. The safety report submitted to CECMED after this 7-day observation period indicated local injection-site reactions as the only adverse effects. With this data confirming the vaccine's safety (already demonstrated in phase 1,2 and partial 3 clinical trials in adults), the second group of 25 children, 3-11 years old, was authorized to receive their vaccinations. To date, this clinical trial has proceeded smoothly. The other vaccine, Abdala, will also enter pediatric clinical trials this year. We are vaccinating our adult population quickly, but they can be carriers of SARSCoV-2 and infect their children at home, which is why we are emphatic about pediatric trials and vaccination.

MEDICC Review: Many developing countries are inspired by Cuba's capacity to produce and authorize a COVID-19 vaccine. But isn't it draining scarce resources?

Olga Lidia Jacobo: Controlling this pandemic has been the highest priority for our country-not only public health authorities, but for our President as well. Obviously, many resources are required to properly conduct clinical trials: syringes, PCR kits, laboratory analysis to measure immunogenicity, reagents for neutralization assays and more. Plus, there's the cost of equipping and staffing all the vaccination sites. At this moment, we are facing a scarcity of certain medicines in Cuba, it's true, but we have those needed to treat COVID-patients.

MEDICC Review: US sanctions and their extraterritorial applications, strengthened under the previous US administration and still in place, certainly don't help...

Olga Lidia Jacobo: Where the US policy affects CECMED's work the most is in our laboratories. Materials, especially reagents, are very difficult to procure. The same holds true for any equipment that breaks: finding replacement parts or buying new equipment becomes especially problematic under the US blockade.

This policy affects every aspect of life in our country-it's not only limited to the inputs and equipment needed in labs. One indispensable part of our work involves printing reports and we've been dangerously low on paper and toner since last year. This gives you an idea of the comprehensiveness of this policy-something as simple as paper is hard for Cuba to purchase on the international market. Building maintenance is another area affected, as well as replacing broken or obsolete computers, which is not only difficult, but costly.

MEDICC Review: It's hard to conceive working under these conditions, with such responsibility, in the midst of a global pandemic. What keeps you going?

Olga Lidia Jacobo: It's a huge challenge, but we have a really good team at CECMED, in terms of responsibility and dedication to the work we're doing. Working together day after day has forged an environment of close collaboration where people are willing to go the extra mile. CECMED also has a solid, stable workforce-sure some people come and go, but on the whole, our staff is committed long-term and that has been an important asset and strength. We wish we could do more, much more actually, but we face certain limitations.

I couldn't do my job without the support of my family. It just would not be possible. I love how we've organized our household so that everyone has their individual duties and responsibilitiesmy husband does the shopping, my daughter cleans the house, I do the laundry and my son and daughter-in-law are in charge of the kitchen-which means I, thankfully, don't have to cook. Family too, is an ever-present strength in this fight against the pandemic. $-1 /$ - 


\section{NOTES AND REFERENCES}

1. Frank M. Cuba says second COVID-19 vaccine Soberana 2 boasts $91.2 \%$ efficacy [Internet]. London: Reuters; 2021 Jul 8 [cited 2021 Jul 9]. Available at: https:// www.reuters.com/business/healthcare-pharmaceuticals/cuba-says-second -covid-vaccine-soberana-2-boasts-912-efficacy-2021-07-09/

2. Valdes-Balbin $Y$, Santana-Mederos $D$, Quintero $L$, Fernández $S$, Rodríguez L, Sanchez-Ramirez B, et al. SARS-CoV-2 RBD-Tetanus Toxoid Conjugate Vaccine Induces a Strong Neutralizing Immunity in Pre-Clinical Studies. ACS Chem Biol [Internet]. 2021 Jul 4 [cited 2021 Jul 7]. Available at: https://pubs .acs.org/doi/10.1021/acschembio.1c00272

3. Previous MEDICC Review articles about Cuba's clinical trial regulatory authority and process include:

http://mediccreview.org/science-at-the-service-of-public-health-rafael-perez -cristia-md-phd-center-for-state-control-of-medicines-and-medical-devices/ https://mediccreview.org/the-abcs-of-clinical-trials-in-cuba/

https://mediccreview.org/covid-19-requires-innovation-regulation-and-rigor-arteaga/

\section{THE AUTHORS}

Tania Lizz Aguilar-Guerra MD MS, embryologist with master's degrees in medical genetics and medical education. Editorial Associate, MEDICC Review. https://orcid.org/0000-0001-5047-2282

Esther María Fajardo-Díaz MS, biologist with a master's degree in biochemistry. Issue Coordinator, MEDICC Review. https://orcid .org/0000-0002-5060-2240

Conner Gorry MA, journalist with a master's degree in international policy. Senior Editor, MEDICC Review. https://orcid.org/0000-0001 $-8212-3386$ 